\title{
Pathogenesis of Diverticulosis and Diverticular Disease
}

\author{
Juozas Kupcinskas ${ }^{1}$, Lisa L. Strate ${ }^{2}$, Gabrio Bassotti ${ }^{3}$, Gabriele Torti ${ }^{3}$, László Herszenyi ${ }^{4}$, Peter Malfertheiner ${ }^{5}$, Claudio \\ Cassieri' $^{6}$, Marjorie M.D. Walker ${ }^{7}$, Antonio Tursi ${ }^{8}$
}

1) Department of

Gastroenterology, Lithuanian

University of Health Sciences,

Kaunas, Lithuania; 2)

Division of Gastroenterology,

Department of Medicine,

Harborview Medical Center,

University of Washington

Medical School, Seattle,

Washington, USA; 3 )

Department of Clinical and

Experimental Medicine,

Gastroenterology and

Hepatology Section, "Santa

Maria della Misericordia"

Hospital, University of

Perugia, San Sisto (PG),

Italy; 4) 2nd Department

of Medicine, Semmelweis

University, H-1088 Budapest,

Hungary; 5) Department of

Gastroenterology, Hepatology

and Infectious Diseases,

Otto-von-Guericke University

Hospital, Magdeburg,

Germany; 6) Division of

Internal Medicine and

Gastroenterology, "Cristo

Re" Hospital, Rome, Italy; 7)

University of Newcastle, Faculty

of Health and Medicine, School

of Medicine \& Public Health,

Australian Gastrointestinal

Research Alliance, Callaghan

NSW 2308, Australia; 8)

Territorial Gastroenterology

Service, ASL BAT, Andria, Italy

\footnotetext{
Address for correspondence:

Antonio Tursi, MD

Via Torino, 49

76123 Andria (BT), Italy

antotursi@tiscali.it
}

\begin{abstract}
In this session different problems regarding the pathogenesis of diverticular disease were considered, including "Genetics", "Neuromuscular function abnormalities", "Patterns of mucosa inflammation", and "Impact of lifestyle". The patients affected by diverticular disease have clear genetic pattern, that might predispose to the occurrence of the disease as well as to its complications. Neuromuscular abnormalities may be recognized already at the stage of diverticulosis, and inflammation may explain symptoms occurrence in symptomatic uncomplicated diverticular disease (SUDD) or symptoms persistence after an episode of acute diverticulitis. Finally, lifestyle might also have an impact on symptoms' occurrence. Specifically smoking, but also obesity seem to play an important role, while the role of low-fiber diet and constipation is now under debate.
\end{abstract}

Key words: diverticular disease - acute diverticulitis - colonoscopy - genetics - fiber - low-grade inflammation - neuromuscular alteration - symptomatic uncomplicated diverticular disease.

Abbreviations: DD: diverticular disease; SCAD: segmental colitis associated with diverticulosis; SUDD: symptomatic uncomplicated diverticular disease.

\section{GENETICS}

Diverticular disease (DD) of the colon is a common disease with an increasing impact for the National Health Systems [1]. It is a complex disorder resulting from an interaction of multiple factors; however, the exact pathogenesis of this disease is not known. Gathered evidence suggests that genetic factors contribute to the development of DD [2]. Convincing data that support the role of genetic factors in DD come from monogenic disorders of connective tissue including Williams-Beuren syndrome, Coffin-Lowry syndrome and Autosomal Dominant Polycystic Kidney Disease where colon diverticula occur at a very early age [2]. A couple of epidemiological twin studies have also recently pointed to the significant role of inherited factors in the development of DD [3, 4]. Several small casecontrol studies have initially attempted to identify certain single nucleotide polymorphisms (SNPs), but were underpowered to provide significant findings $[5,6]$. A larger case control study including 422 patients with diverticulosis and 285 controls found that variant rs3134646 in COL3A1 gene is associated with the risk of developing colonic diverticulosis in men [7]. However, the most important data on genetic predisposition of DD come from three very recent genome-wide association studies (GWAS) [7-9]. The first GWAS on Iceland population showed that variants in ARHGAP15 and COLQ genes were linked with uncomplicated DD and variants in FAM155A were associated with diverticulitis [8]. Additionally, 37 susceptibility loci with genome-wide significance were identified in a recent study from Maguire et al. [9] with replication of 8 loci. The largest GWAS study to date employed UK Biobank and imputed genotypes using 31964 cases and 419135 controls [10]. These associations were then replicated in a European sample of 3893 cases and 2829 diverticula-free controls and evaluated for risk contribution to diverticulitis and uncomplicated diverticulosis and identified 48 genetic risk loci [10]. The genetic data from all three GWAS studies clearly show that DD primarily is a disorder of intestinal neuromuscular function and of impaired connective fibre support, while an additional diverticulitis risk might be conferred by epithelial dysfunction related genes [8-10]. To date, there are no studies evaluating how identified 
genetic risk factors could serve for clinical decisions making in $\mathrm{DD}$ prevention or management and these subjects are topics for future research.

\section{NEUROMUSCULAR FUNCTION ABNORMALITIES}

It has been hypothesized that in colonic DD higher intraluminal pressures in the affected segments may contribute to the production of pulsion diverticula [11]. However, data from rectosigmoid motor activity between controls and patients with DD were not univocal [12]. Further information was obtained by recording whole colonic motor activity for prolonged periods of time (24 hours or more) [13], and assessing visceral perception and compliance in the rectum and rectosigmoid area [14].

\section{Colonic motility}

In a previous study we recorded 24-h colonic motility in patients with asymptomatic DD and in healthy controls; patients displayed significantly increased motility in the segments with diverticula [15], an abnormal motor response to eating, and a significant increase of high-amplitude propagated contractions. In another 24-h study in patients with SUDD, compared to controls, patients had a significant increase of regular contractile patterns, with more than $80 \%$ represented by $2-3$ cycles per minute pattern. More than one third of these patients (none of the controls) reported associated cramping lower abdominal pain during a regular contractile pattern [16].

\section{Colonic sensory activity}

By comparing data from symptomatic, asymptomatic patients with DD and controls, rectal perception of distention was increased in symptomatic patients compared to the other groups; rectal compliance curves were not different between the three groups [17]. In the sigmoid colon, perception was increased in symptomatic, but not in asymptomatic patients (before and after meals) compared to controls, with similar compliances in the three groups. The colonic response to eating was not different between groups. Thus, patients with symptomatic disease display increased perception of distention in both the affected (sigmoid) segment and in the unaffected rectum; this increase of perception is not due to an altered wall compliance. A further study showed visceral hypersensitivity in symptomatic patients possibly mediated by ongoing low-grade inflammation and upregulation of tachykinins [18].

In conclusion, neuromuscular dysfunction plays a paramount role in $\mathrm{DD}$, but more investigations are needed to establish the true role of this dysfunction.

\section{PATTERNS OF COLONIC MUCOSAL INFLAMMATION}

Diverticulosis, namely the presence of colonic diverticula, is a common condition. In a recent population study $17.5 \%$ of participants had diverticula and the sigmoid was always involved [19]. An endoscopy study of all comers demonstrated $32.6 \%$ had diverticula, $71.4 \%$ of those aged $\geq 80$ years [20]. The presence of inflammation, diverticulitis - macroscopic inflammation of diverticula with related acute or chronic complications is seen in $4 \%$ of those with diverticulosis (with an 11-year follow up) and $11 \%$ in younger patients (40-49 yrs) [20]. In the patients with acute inflammatory complications of DD, inflammation in the mucosa is evident and acute in nature. When complications ensue and diverticula break, an acute abdomen leads to surgical intervention and consequent surgical pathology shows full thickness and peritoneal inflammation. Segmental colitis associated with diverticula (SCAD), a unique form of mucosal or full thickness inflammation that looks similar with IBD, is observed in $11 \%$ of those with diverticulosis and $2 \%$ of patients investigated by colonoscopy [21-23]. This is a specific mucosal inflammatory process, confined to the sigmoid and descending colon. In SUDD, the site of inflammation is likely deep in the muscularis and may interact with nerves, as patients exhibit visceral hypersensitivity, and this may be mediated by ongoing low grade inflammation and upregulation of tachykinins [18]. However, in a community population with DD, there was no association between symptomatic diverticulosis (abdominal pain or diarrhoea) and serological (C-reactive protein) or mucosal inflammation - the inflammatory gradient was intact [19]. In small studies, macrophages have been shown to be increased in SUDD in 8 patients [24] and also lymphocytes were elevated in 10 patients [25], but when assessing every aspect of colon pathology in a community population of 127 subjects [21] and in an endoscopy population of 255 patients [26] no evident mucosal inflammation was noted.

DD shows a spectrum of pathological changes, which can be mucosal and/or neuromuscular. Other disorders may co-exist and the key to unravelling pathogenesis is in identifying triggers to inflammation. These include an aging gut, neuromuscular disorders, disordered immune reactivity and dysbiosis, genetics, diet, lifestyle and smoking [27].

\section{IMPACT OF LIFESTYLE}

Diverticular disease is called a disease of Western Cultures because of the rapid and continuous increase in incidence following the Industrial Revolution that has been largely attributed to changes in diet and lifestyle. Studies consistently indicate that low dietary fiber is associated with risk of incident diverticulitis [28,29], although the association between fiber and asymptomatic diverticulosis is uncertain [30,31]. In a large UK cohort, individuals who consumed more than 25 grams of fiber had a $40 \%$ decreased risk of hospitalization for diverticulitis [28], but two cross-sectional colonoscopy studies found no association between fiber intake and asymptomatic diverticulosis [30,31]. Physical activity, especially vigorous activity, also decreases the risk of diverticulitis [32]. On the other hand, red meat consumption is positively associated with risk of diverticulitis (relative risk 1.2 for each serving of red meat) [33]. When considering diet as a whole, a Western dietary pattern (high in red meat and refined grains), increases risk of diverticulitis, whereas a prudent pattern (high in fruits, vegetables and whole grains) decreases risk [34]. Obesity, and central obesity in particular, is associated with risk of diverticulitis. In a prospective study, men with a body mass index $(\mathrm{BMI})>30 \mathrm{~kg} / \mathrm{m} 2$ had a relative risk of 1.8 [35]. Weight 
gain also contributes to the risk of diverticulitis [36]. Smoking is another risk factor especially for complicated diverticulitis $(37,38)$. The importance of diet and lifestyle is demonstrated in a study that found that men who adhered to five healthy lifestyle factors (BMI $18-25 \mathrm{~kg} / \mathrm{m} 2$, fiber intake $>23 \mathrm{~g} /$ day, red meat $<4$ servings/week, two hours of exercise/ week and no smoking had a $75 \%$ decreased diverticulitis risk compared to men who did not adhere to any healthy lifestyle factors [39].

\section{REFERENCES}

1. Papa A, Papa V. The Economic Burden of Diverticular Disease. J Clin Gastroenterol 2016;50 Suppl 1:S2-S3. doi:10.1097/ MCG.0000000000000598

2. Tursi A. Current and Evolving Concepts on the Pathogenesis of Diverticular Disease. J Gastrointestin Liver Dis 2019;28:225-223. doi:10.15403/JGLD-184

3. Granlund J, Svensson T, Olén O, et al. The genetic influence on diverticular disease--a twin study. Aliment Pharmacol Ther 2012;35:1103-1107. doi:10.1111/j.1365-2036.2012.05069.x

4. Strate LL, Erichsen R, Baron JA, et al. Heritability and familial aggregation of diverticular disease: a population-based study of twins and siblings. Gastroenterology 2013;144:736-742.e1. doi:10.1053/j. gastro.2012.12.030

5. Connelly TM, Berg AS, Hegarty JP, et al. The TNFSF15 Gene Single Nucleotide Polymorphism rs7848647 Is Associated With Surgical Diverticulitis. Ann Surg 2014;259:1132-1137. doi: 10.1097/ SLA. 0000000000000232

6. Beasley WD, Beynon J, Jenkins GJ, Parry JM. Reprimo $824 \mathrm{G}>\mathrm{C}$ and p53R2 $4696 \mathrm{C}>\mathrm{G}$ single nucleotide polymorphisms and colorectal cancer: a case-control disease association study. Int J Colorectal Dis 2008;23:375-381. doi:10.1007/s00384-007-0435-3

7. Reichert MC, Kupcinskas J, Krawczyk M, et al. Variant of COL3A1 (rs3134646) Is associated with risk of developing diverticulosis in white men. Dis Colon Rectum 2018;61:604-611. doi:10.1097/ DCR.0000000000001001

8. Sigurdsson S, Alexandersson KF, Sulem P, et al. Sequence variants in ARHGAP15, COLQ and FAM155A associate with diverticular disease and diverticulitis. Nat Commun 2017;8:15789. doi:10.1038/ ncomms 15789

9. Maguire LH, Handelman SK, Du X, Chen Y, Pers TH, Speliotes EK. Genome-wide association analyses identify 39 new susceptibility loci for diverticular disease. Nat Genet 2018;50:1359-1365. doi:10.1038/ s41588-018-0203-Z

10. Schafmayer C, Harrison JW, Buch S, et al. Genome-wide association analysis of diverticular disease points towards neuromuscular connective tissue and epithelial pathomechanisms. Gut 2019;68:854865. doi:10.1136/gutjnl-2018-317619

11. Parks TG, Connell AM. Motility studies in diverticular disease of the colon. Gut 1969;10:534-542. doi:10.1136/gut.10.7.534

12. Leandro PA, Cecconello I, Habr-Gama A, de Olivereira e Silva A, Pontes JF. Gastrointestinal motility in normal subjects and patients with diverticulosis of the colon. Arq Gastroenterol 1984;21:157-163.

13. Bassotti G, Crowell MD, Whitehead WE. Contractile activity of the human colon: lessons from 24 hour studies. Gut 1993;34:129-133. doi:10.1136/gut.34.1.129

14. Camilleri M, Bharucha AE, di Lorenzo C, et al. American Neurogastroenterology and Motility Society consensus statement on intraluminal measurement of gastrointestinal and colonic motility in clinical practice. Neurogastroenterol Motil 2008;20:1269-1282. doi:10.1111/j.1365-2982.2008.01230.x

15. Bassotti G, Battaglia E, Spinozzi F, Pelli MA, Tonini M. Twenty-four hour recordings of colonic motility in patients with diverticular disease: evidence for abnormal motility and propulsive activity. Dis Colon Rectum 2001;44:1814-1820. doi:10.1007/bf02234460

16. Bassotti G, Battaglia E, De Roberto G, Morelli A, Tonini M, Villanacci V. Alterations in colonic motility and relationship to pain in colonic diverticulosis. Clin Gastroenterol Hepatol 2005;3:248-253. doi:10.1016/ s1542-3565(04)00614-7

17. Clemens CH, Samsom M, Roelofs J, van Berge Henegouwen GP, Smout AJ. Colorectal visceral perception in diverticular disease. Gut 2004;53:717-722. doi:10.1136/gut.2003.018093

18. Humes DJ, Simpson J, Smith J, et al. Visceral hypersensitivity in symptomatic diverticular disease and the role of neuropeptides and low grade inflammation. Neurogastroenterol Motil 2012;24:318-e163. doi:10.1111/j.1365-2982.2011.01863.x

19. Järbrink-Sehgal ME, Rassam L, Jasim A, et al. Diverticulosis, Symptoms and Colonic Inflammation: A Population-Based Colonoscopy Study. Am J Gastroenterol 2019;114:500-510. doi:10.14309/ajg.0000000000000113

20. Everhart JE, Ruhl CE. Burden of digestive diseases in the United States part II: lower gastrointestinal diseases. Gastroenterology 2009;136:741754. doi:10.1053/j.gastro.2009.01.015

21. Tursi A, Elisei W, Giorgetti GM, Aiello F, Brandimarte G. Inflammatory manifestations at colonoscopy in patients with colonic diverticular disease. Aliment Pharmacol Ther 2011;33:358-365. doi:10.1111/j.13652036.2010.04530.x

22. Goldstein NS, Leon-Armin C, Mani A. Crohn's colitis-like changes in sigmoid diverticulitis specimens is usually an idiosyncratic inflammatory response to the diverticulosis rather than Crohn's colitis. Am J Surg Pathol 2000;24:668-675. doi:10.1097/00000478-200005000-00005

23. Lanas A, Abad-Baroja D, Lanas-Gimeno A. Progress and challenges in the management of diverticular disease: which treatment? Therap Adv Gastroenterol 2018;11:1756284818789055. doi:10.1177/1756284818789055

24. Barbara G, Scaioli E, Barbaro MR, et al. Gut microbiota, metabolome and immune signatures in patients with uncomplicated diverticular disease. Gut 2017;66:1252-1261. doi:10.1136/gutjnl-2016-312377

25. Tursi A, Brandimarte G, Elisei W, et al. Assessment and grading of mucosal inflammation in colonic diverticular disease. J Clin Gastroenterol 2008;42:699-703. doi:10.1097/MCG.0b013e3180653ca2

26. Peery AF, Keku TO, Addamo C, et al. Colonic Diverticula Are Not Associated With Mucosal Inflammation or Chronic Gastrointestinal Symptoms. Clin Gastroenterol Hepatol 2018;16:884891.e1. doi:10.1016/j.cgh.2017.05.051

27. Strate LL, Modi R, Cohen E, Spiegel BM. Diverticular disease as a chronic illness: evolving epidemiologic and clinical insights. Am J Gastroenterol 2012;107:1486-1493. doi:10.1038/ajg.2012.194

28. Crowe FL, Appleby PN, Allen NE, Key TJ. Diet and risk of diverticular disease in Oxford cohort of European Prospective Investigation into Cancer and Nutrition (EPIC): prospective study of British vegetarians and non-vegetarians. BMJ. 2011;343:d4131. doi:10.1136/bmj.d4131

29. Crowe FL, Balkwill A, Cairns BJ, et al. Source of dietary fibre and diverticular disease incidence: a prospective study of UK women. Gut 2014;63:1450-1456. doi:10.1136/gutjnl-2013-304644

30. Peery AF, Barrett PR, Park D, et al. A high-fiber diet does not protect against asymptomatic diverticulosis. Gastroenterology 2012;142:266272.e1. doi:10.1053/j.gastro.2011.10.035 
31. Peery AF, Sandler RS, Ahnen DJ, et al. Constipation and a low-fiber diet are not associated with diverticulosis. Clin Gastroenterol Hepatol 2013;11:1622-1627. doi:10.1016/j.cgh.2013.06.033

32. Strate LL, Liu YL, Aldoori WH, Giovannucci EL. Physical activity decreases diverticular complications. Am J Gastroenterol 2009;104:1221-1230.

33. Cao Y, Strate LL, Keeley BR, et al. Meat intake and risk of diverticulitis among men. Gut 2018;67:466-472. doi:10.1136/gutjnl-2016-313082

34. Strate LL, Keeley BR, Cao Y, Wu K, Giovannucci EL, Chan AT. Western Dietary Pattern Increases, and Prudent Dietary Pattern Decreases, Risk of Incident Diverticulitis in a Prospective Cohort Study. Gastroenterology 2017;152:1023-1030.e2. doi:10.1053/j. gastro.2016.12.038

35. Strate LL, Liu YL, Aldoori WH, Syngal S, Giovannucci EL. Obesity increases the risks of diverticulitis and diverticular bleeding. Gastroenterology 2009;136:115-122.e1. doi:10.1053/j. gastro.2008.09.025

36. Ma W, Jovani M, Liu PH, et al. Association Between Obesity and Weight Change and Risk of Diverticulitis in Women. Gastroenterology 2018;155:58-66.e4. doi:10.1053/j.gastro.2018.03.057

37. Hjern F, Wolk A, Hakansson N. Smoking and the risk of diverticular disease in women. Br J Surg 2011;98:997-1002. doi:10.1002/bjs.7477

38. Humes DJ, Ludvigsson JF, Jarvholm B. Smoking and the Risk of Hospitalization for Symptomatic Diverticular Disease: A PopulationBased Cohort Study from Sweden. Dis Colon Rectum 2016;59:110-114. doi:10.1097/DCR.0000000000000515

39. Liu PH, Cao Y, Keeley BR, et al. Adherence to a Healthy Lifestyle is Associated With a Lower Risk of Diverticulitis among Men. Am J Gastroenterol 2017;112:1868-1876. doi:10.1038/ajg.2017.398 